\title{
Toward using Business Process Intelligence to Support Incident Management Metrics Selection and Service Improvement
}

\author{
Bianca Trinkenreich, Valdemar T. F. Confort, Gleison Santos, Flávia Maria Santoro \\ Department of Computing \\ Universidade Federal do Estado do Rio de Janeiro (UNIRIO) \\ Rio de Janeiro, RJ, Brazil \\ \{bianca.trinkenreich, valdemar.confort, gleison.santos, flavia.santoro\}@ uniriotec.br
}

\begin{abstract}
Background: Businesses are increasingly dependent on IT services, and providers need to deliver fast, with high quality and low cost. An incident is an event that can lead to loss or disruption of services. Incident management reinstates normal service operation as quickly as possible and mitigates negative impact to business, ensuring agreed levels of service quality. So, reduce resolution time is usually the most important goal for incidents. Aims We aim to obtain knowledge about process and identify adequate metrics for Incident Management to help reduction of resolution time. Our research questions are: (i) Which Incident Management sub-process is causing more impact to resolution time? (ii) Which metrics can be used to measure this sub-process? (iii) What actions can be taken to improve Incident Management process in order to reduce impact of this subprocess in resolution time? Method: We present a case study in a global large company that considers reduction of incidents resolution time as a goal. Results: By applying BPM, BPI and Process Mining we were able to discover the underlined process and a bottleneck for resolution time. Moreover, we proposed metrics to improve process and service quality by applying GQM+Strategies.
\end{abstract}

Keywords: Measurement, Metric, Incident Management, BPI, Process Mining

\section{INTRODUCTION}

Service is about delivering value to customers by facilitating results they want to achieve without taking costs and risk ownership. Many organizations started to outsource IT in order to build services capabilities to its products, being able to provide faster and more accurate service to customers [22].

IT service management is a set of specialized organizational capabilities for providing value to customers through services. Its practice has been growing by adopting an IT management service-oriented approach to support applications, infrastructure and processes [10]. In software engineering approach, while software development delivers a product, software maintenance provides service to customers, as it modifies a software system or component after delivery to correct faults, improves performance or other attributes, or

(DOI reference number: 10.18293/SEKE2015-110) adapts to a changed environment. Accepting software maintenance as a service perspective, it should follow IT service management and be developed and improved as a service either [23].

Guidance on how to develop and improve IT service maturity practices is a key factor to improve service performance and customer satisfaction [8]. CMMI-SVC (Forrester et al., 2010) model was created to attend this need and it is based on traditional models like ITIL [10] and international standards as ISO/IEC 20000 [11]. This model requires the identification of appropriate metrics in order to monitor the various processes executed for service delivering to customers. Business Process Management (BPM) [1] is also used to support processes improvement and governance initiatives. BPM recognizes Information Technology (IT) as one of its pillars [1] and has broad application in business areas. We perceive convergence between both areas. However, references of BPM techniques supporting CMMI-SVC are not common. Having an adequate metrics selection is one of key success for measurement initiatives. In BPM, Business Process Intelligence (BPI) and Process Mining [4] provide solutions on how to support knowledge management of a business process. Furthermore, we can evaluate a process, verify which subprocess is causing more impact to process results and also to support selection of metrics associated to this sub-process, using BPI techniques.

We had selected Incident Management process inspired by Business Process Intelligence Challenge 2013 and 2014 [7], recognizing that it produces a large amount of log data that can drive knowing users' needs and issues, being a process that is also used for corrective software maintenance support, a process that is mostly considered by service providers for measurement initiatives [20], and the one that, when properly used as a strategic asset [15], can help to provide answers about the service quality [8]. Like that, we are also converging two major fields: Quality of Service and IT Service Maturity Models, and BPM and Process Mining.

This paper presents an approach to support Measurement and Maturity Models on selection of Incident Management metrics to attend organization goals by using BPM, BPI and 
Process Mining. Besides this introduction, in Section 2 we present a theoretical framework, in Section 3 we present related works, in Section 4 we present the case study and finally our final considerations in Section 5.

\section{2 THEORETICAL FRAMEWORK}

\section{A. IT Service Quality, Maturity Models and Measurement}

A service is an intangible and non-storable deliver customer value way, making it easier to get results without needing to be responsible for risks and costs associated with the work [8] [9]. Guidance on how to develop and improve service maturity is a key factor for service provider performance and customer satisfaction. In order to be able to offer quality, the supplier must continually assess the way service is being provided and what the customer expects. A customer will be unsatisfied with IT service providers who occasionally exceed expectations but at other times disappoint. Providing consistent quality is important, but is also one of the most difficult aspects of software and service industry [11].

Maturity models focus on improving organizations processes due to the assumption that product or system quality is highly influenced by the quality of process used to develop and keep it. Through essential elements of effective processes and an evolutionary path for improvement, maturity models provide guidelines on how to design processes, as an application of principles to meet the endless cycle of process improvement [8].

CMMI-SVC [8] is a service maturity model based on CMMI concepts and practices and other standards and service models, such as ITIL [10], COBIT [12], ISO/IEC 20000 [11], and ITSCMM [13]. CMMI-SVC is composed of 24 process areas and 5 maturity levels (from 2 to 5 ), and includes required activities to create, deliver and manage services [8]. Maturity levels are used as the obtained classification in assessments, and also as a recommended evolutionary path for organizations intending to improve their processes in order to provide better services. Although CMMI-SVC is not intended to be used only by IT firms, those organizations certainly can benefit of it. Measurement is included since CMMI initial levels. Due to the financial difficult to measure and control all existing processes, selected measures must be aligned to organizational business goals in order to provide effective information for decision making. This choice is not trivial [9]. GQM paradigm (Goal Question Metric) is generally used to select metrics to provide expected visibility of the organization's strategic objectives. Some criteria for selecting metrics are: relationships with strategic objectives, coverage of lifelong service, availability and objectivity frequency that data can be collected, changing sensitivity, performance visibility and representation [8].

GQM+Strategies approach [21] is an extension of the GQM, and takes strategies and KPIs as input for a model from the business level down to project and operational levels and back up. GQM+Strategies focuses on filling organizations vertical gaps and help creation of objectives and strategies and deriving metrics that are aligned with high-level business goals and also providing a mechanism to monitor success and failure of goals and strategies through measurement.
This approach has two core components: a process and a model, and introduces the idea of having multi-level goals, strategies, context, assumptions, and a broader interpretation model. The process includes generating a grid that represents the hierarchy of operational, strategies, measures and interpretations models, planning, executing and evaluating those strategies with recommendations for future improvements. The model is called "grid", which is a comprehensive model with a notation to support organizations on developing their operational and measureable business goals, selecting strategies to implement them, providing communication about those goals and strategies to stakeholders of organization, and then deriving those goals into aligned sets of other lower-level goals and strategies. Through the grid, all parts of the organization can recognize their role on reaching top level goals, how it can be measured and how results are interpreted [21].

GQM+Strategies approach basic concepts are about Organization goals (what organization wants to achieve to reach its objectives), Strategies (how to achieve those goals), Context Factors (external and internal environments), Assumptions (unknown estimations) and GQM graphs (how to measure if goal was reached and strategy was successful or failed) [21].

\section{B. Incident Management}

According to ITIL [10], an incident is defined as an unplanned interruption or quality reduction while providing IT services. For example, a failure or hardware configuration that has not yet impacted software or services is also considered incident. Incident Management deals with all types of incidents, including failures or questions made by users or automatically detected by monitoring tools.

Incidents are events that, if not addressed, can eventually cause the supplier to fail in meeting its service level agreement (SLA) [8]. SLA is an agreement between service provider and customer, describing the service that will be provided, documenting service levels goals, and each involved part responsibilities. A SLA failure can cause contractual penalties to be applied [4]. Service Desk is the first support level, the team that receives users requests and process according defined flow, providing solution using knowledge articles information, or when is not possible to solve the issue or attend the request, assigning incident ticket for next levels support.

In CMMI-SVC [8], Incident Resolution and Prevention (IRP) is the level 3 process area that handles incidents solution and prevention through problems analyzing and treatment. IRP activities include: identify, analyze, assign, monitor status and progress, escalate if needed, and implement workarounds to restore service, communicate progress, validate resolution with stakeholders and analyze root cause of incidents.

Avoiding overflow within the SLA incident response is usually one of the strategic goals of service providers who pay penalty for noncompliance. Knowing the behavior patterns of Incident Management process allows the identification of which sub-processes are not performing well and needs to be controlled and improved. 


\section{Process Intelligence}

Business Process Intelligence (BPI) refers to the application of Business Intelligence techniques (BI) to business processes [2] [3]. Typically, data sources come from Business Process Management Systems (BPMS) and Process-Aware of Information Systems (PAIS). Recording business events that occur during the execution of processes are called Event Logs.

Event log reveals important insights on how a process actually works. Here we look at more specific BPI role, identifying three major scenarios for application event log usage: automatic process discovery, performance analysis and verification of conformity [1]. The first is about the way process is implemented in practice; the second provides a discussion about operation cost and time and the third is to verify if a set of rules is being followed. Process mining is an enabling technology for CPM (Corporate Performance Management), BPI (Business Process Intelligence), TQM (Total Quality Management), Six Sigma and others [4]. However, mining processes from an event $\log$ is not a trivial task. A processes mining project can be developed in five stages [4]. Figure 1 reproduces this cycle.

In stage 0 , project is justified and planned. In stage 1 , event data, models, objectives and questions are extracted. At this point, a domain expert presence is important to define issues and assist in data understanding. Stage 2 takes as input the output of previous stage and focuses on producing an event log and a control flow model connected to this event log. In this phase, process discovery techniques can be applied. With a relatively structured process, other perspectives (date, time, resource) can be evaluated during stage 3 . As a result of step 3, a process model will be discovered and an event log will be used to provide operational support. In stage 4, knowledge extracted from previous stage is combined with running cases, providing chance for interventions, predictions and recommendations. We stand out that stages 3 and 4 can only be achieved if the process is sufficiently stable and structured [4].

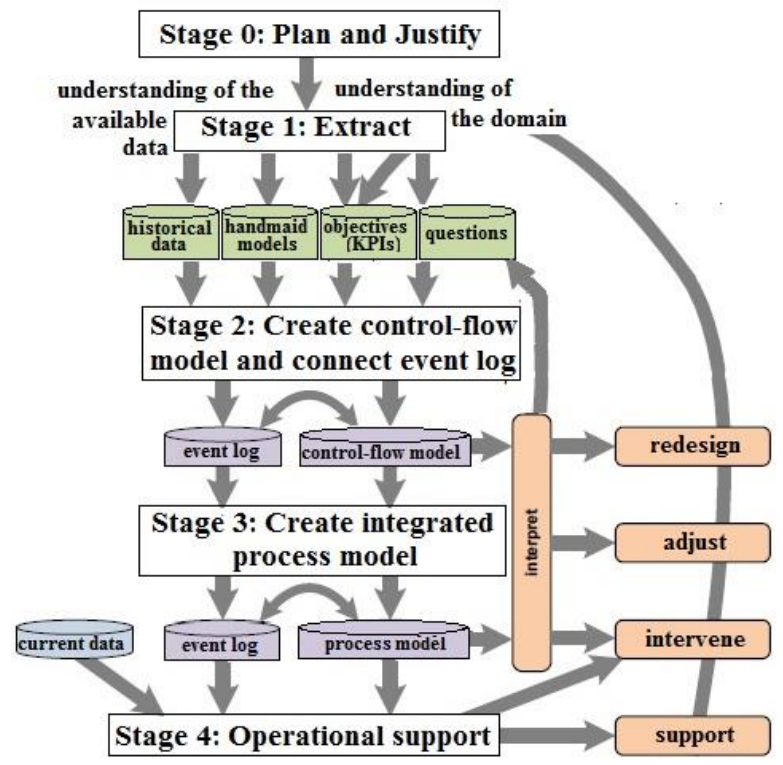

Figure 1 - Life cycle showing a mining process project stages [4].

\section{RELATED WORKS}

The scope of this paper is applying BPI techniques to a real incidents activities $\log$ in order to discover the Incident Management process and select its sub-processes that are more influencing results. This differs importantly from the field of root cause analysis because we are not investigating why incidents are occurring, but how long they take to be solved and which activity is causing more impact and could further be analyzed in order to improve incidents solving time. Nevertheless, much of our related work comes from incidents analysis and repairing. Following paragraphs show how our investigation and exploratory study had been inspired by methods and results from this area, even though no previous work precisely shares our scope.

Franke et al. [16] use incidents logs from 1.800 incidents in a large Nordic bank to find statistical distribution of IT service time to recovery. Authors show that log-normal distribution can help to understand what the best fit of IT service time to recovery, predicting downtime and costs to be used by organizations would be. This article does not use activities log to find what sub-process is taking more time to happen and impacting total process results and does not use Business process intelligence (BPI) for analysis neither.

Both 2013 and 2014 International Business Process Intelligence Challenges were about analyzing logs activities through process mining [7]. 2013 edition was about Incidents and Problems and 2014 edition was about finding correlation between Changes and Incidents.

Ferreira and Rabuge [17] had presented a methodology to apply business process analysis in a hospital emergency service to identify regular behavior, process variants, and exceptional medical cases. Ferreira and Silva [27] had presented another case study to determine how far the current incident management process is from the best practices described by ITIL in order to draw requirements for the new system. Although they also use process mining to extract the behavior of the existing process, they do not use it to discover subprocesses and which part is impacting the most the results in order to support measures selections for IT services maturity model. Also, they had used ProM tool [5], we had used Disco tool [14].

We could also notice a growing body of knowledge about case studies in different application domains regarding to describe reverse engineering with process mining from event logs. Mieke et al. [18] had analyzed procurement process to understand about internal fraud risk reduction.

\section{CASE STUDY}

A case study method is an exploratory research technique used to highlight and explore aspects, which may guide providing directions for the question. This method is relevant for information system when researcher can study it in a real environment, and allows answering "how" and "why" questions. Although this paper scope is measurement, which is a quantitatively approach, a case study is commonly used as a qualitative method for researching information systems [19]. The research process we had used is explained as follows. 


\section{A. Case Study Planning}

In order to execute the case study, we followed a set of stages depicted in Figure 2. Research questions that we aim to answer are: (i) Which Incident Management sub-process is causing more impact to resolution time? (ii) Which metrics can be used to measure and control this sub-process? (iii) What actions can be taken to improve Incident Management process in order to reduce impact of this sub-process in resolution time?

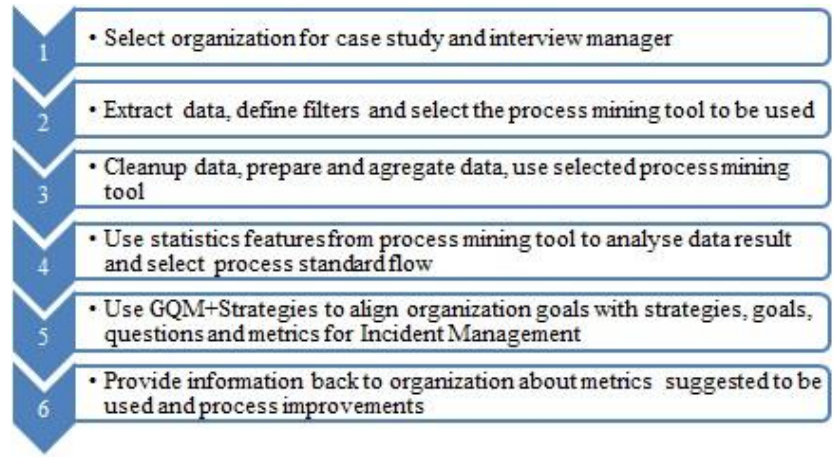

Figure 2 - Case study stages

\section{B. Case Study Execution}

In first stage, we identified an organization to perform the case study: Organization A is a large global organization headquartered in Brazil. It operates in over 30 countries and has offices, operations, exploration and joint ventures across five continents. The case study was performed on its IT services infrastructure department. One of the researchers that conducted the case study works there on improving quality of services and other researcher is a BPM and BPI specialist.

The IT Services Department provide IT services for all other departments of the organization following ITIL library practices [10], but it is not certified by any software or services maturity model. Incident Manager was interviewed and informed that he spends lots of effort to perform, analyze, report and plan new metrics in order to attend organizational goal of reducing incidents resolution time. Also, he had mentioned that Organization A uses only three Incident Management metrics, that indirectly should support attending resolution time: "Incidents resolved within target" (number of closed incident requests that were in accordance to service level agreement time), "Service desk resolutions" (number of incidents that were both registered and resolved by first level support without the assistance from another team) and "Incidents backlog" (number of not solved incidents). However, he pointed out that only these metrics are not effective and enough to provide results of reducing resolution time.

We had initiated the second stage by extracting and validating data availability through Organization A Incident Management tool (HP Service Manager 9 - SM9). Our main challenge was to understand SM9 format of event log data. Our effort was reduced by having help from Incident Manager. We had performed a preliminary evaluation to check if extracted data was compatible with defined process mining tools selected to use. Two tools were considered eligible as able to transform activity history on default event log XES [6]: ProM [5] and
Disco [14]. We started using both tools in order to select the most appropriated for considered scenario. We decided to use data for one month (April 2014), due to the huge amount of data, and one application (Intranet), because Incident Manager reported that it is the application that concentrates larger amount of incidents being opened by users.

Because there was no preconceived design process, we conducted a first execution to discover Incident Management process itself and answer some basic questions about it: how many incidents were opened in a determined month, what are the minimum, maximum and mean time closing an incident and which possible flows an incident resolution can have.

ProM tool [5] could not recognize control flow with some instances and this was the first issue found about it. Disco tool [14] could discover control flow without removals or adjustments to original data. Also, Disco tool abstracts its algorithm for process detection, and it resulted in lower effort. Because of those two difficulties about ProM tool, we had chosen to continue with Disco tool.

In third stage we had filtered incidents selected period and application, and started to prepare data. One incident has many activities and events, representing incident lifecycle. Original $\log$ file had 14.815 events. From those, there were 120 different activities. After extraction, we had to perform some cleanup of wrong entries that were not representing real activity names, for example concatenating an activity name with an incident number in same field. Possibly it was because some kind of bug in SM9 extraction tool, but as they represented only $1 \%$ of total, we removed and not considered entire incident registry for those. So, we had excluded $1 \%$ of incidents from data because of what we had considered bad data. After removing them by using Disco tool [14] functionality to filter undesired activities, we could get a process with 27 activities. Through analysis of process variants, we found 507 different paths, from 993 total identified process instances. Although it answers one of basic questions (which flows can an incident resolution has?), at this point this high number of activities and transitions did not allowed us to answer research question (i).

The control flow model identified by Disco tool [14] at this point had a lot of activities and transitions. At this stage, we used a Disco tool native functionality to aggregate some activities and transitions. For example, there are flow cases that go from activity A to activity B and then activity $\mathrm{C}$. There are also cases where activity A flows directly to activity C. Disco tool does easily abstract these two types of cases making a control flow that considers only transition from $\mathrm{A}$ to $\mathrm{C}$. Therefore, we can choose where to drill-down from a general and major flow to a detailed one.

For this research, we considered the most regular flow (with 5 steps) and used Disco statistics and performance features to analyze amount of elapsed time of each transition, in order to help us answering research question (i).

In fourth stage, we had used global statistics feature to identify the amount of total events (11,203 events) and answer basic questions: How many incidents were opened and what are the minimum, maximum and mean time closing an incident. Total of 993 incidents had been opened, with 5.5 days 
of mean and 4.7 days of median to be solved. Minimum time to close an incident was 22 minutes and maximum had lasted 36 days. Even though, as we have shown that most cases had been solved with only 5 activities (Figure 3 ), this was considered a standard for analysis. Based on this, we had simplified incidents flow to a 5-steps process: Open, Assignment, Start Work in Progress, Resolved and Closed.

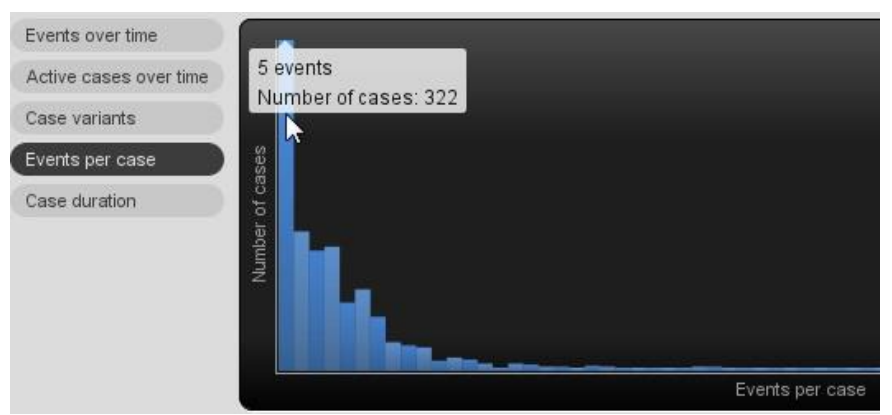

Figure 3 - Graph from Disco tool [14]

Table 1 provides time performance analysis for each transition of the considered 5-steps process. We can notice that it is taking more for someone to take responsibility to solve the incident (Open to Assessment) than to properly solve it (Work in Progress to Resolved), answering research question (i) "Which Incident Management sub-process is causing more impact to resolution time?"

TABLE I. DURATION ANALYSIS FOR EACH TRANSITION

\begin{tabular}{|c|c|c|c|c|}
\hline Transition & $\begin{array}{c}\text { Total } \\
\text { Duration }\end{array}$ & $\begin{array}{c}\text { Max } \\
\text { Duration }\end{array}$ & $\begin{array}{c}\text { Mean } \\
\text { Duration }\end{array}$ & $\begin{array}{c}\text { Median } \\
\text { Duration }\end{array}$ \\
\hline Open $\rightarrow$ Assignment & 68,6 days & 26,3 hours & $\begin{array}{c}109,3 \\
\text { minutes }\end{array}$ & $\begin{array}{c}51,5 \\
\text { minutes }\end{array}$ \\
\hline $\begin{array}{c}\text { Assignment } \rightarrow \text { Work } \\
\text { in Progress }\end{array}$ & 11,6 days & 5,9 days & $\begin{array}{c}24,6 \\
\text { minutes }\end{array}$ & $\begin{array}{c}68,5 \\
\text { seconds }\end{array}$ \\
\hline $\begin{array}{c}\text { Work in Progress } \rightarrow \\
\text { Resolved }\end{array}$ & 13,5 days & 22,4 hours & $\begin{array}{c}49,2 \\
\text { minutes }\end{array}$ & $\begin{array}{c}11,4 \\
\text { minutes }\end{array}$ \\
\hline Resolved $\rightarrow$ Closed & 111 months & 4 days & 3,8 days & 4 days \\
\hline
\end{tabular}

In fifth stage we used GQM+Strategies [21] to align goals, strategies, questions and metrics (Figure 4) in order to suggest a measurement improvement for Organization A, and answer research question (ii) "Which metrics can be used to measure and control this sub-process?". We have used root cause analysis, found that Assignment is taking much time because service desk commit assignment errors, taking longer to define correct team to send incident, which happens because lack of available, correct and updated information. Considered context factor for GQM+Strategies was cost reduction scenario that Organization $\mathrm{A}$ is facing. Considered assumptions for GQM+Strategies was there is already human resources available and with enough expertise to generate information and update knowledge articles. New metrics suggested were: "Time to Own" (number of minutes that an incident is taking to be assigned to correct team), "Incident Assignment Correctness" (percentage of incidents that were assigned to correct team) and "Articles not updated" (number of times that service desk cannot find required information to solve an incident or assign to correct team).

In sixth stage we proposed process improvements for
"Open to Assignment" part of Incident Management process. Knowledge articles used by first level support represent the way that service desk team is able to solve incidents by itself and also assign to proper higher support teams when cannot be solved in first level. So, improve these artifacts is a way to make first level capable of solving more incidents and also reduce time and errors in Assignment phase, which is the bottleneck for incident resolution time (and reducing is the organization goal). Knowledge articles should contain direct, simple and proper questions for a first level support analyst to do when a user calls reporting an error or requesting a service. Search mechanisms should provide easy finding of articles by many key words.

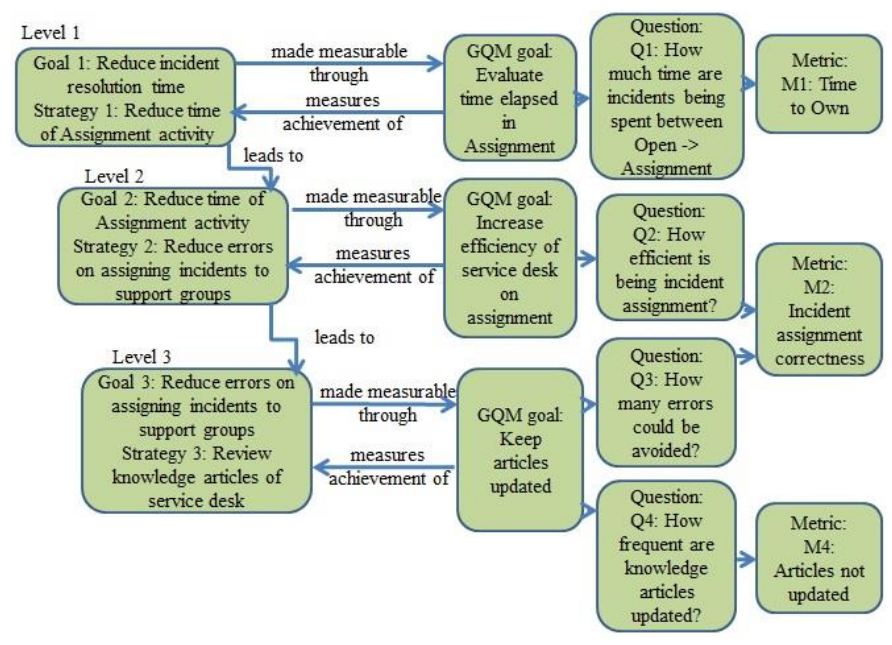

Figure 4 - GQM+Strategies diagram proposed for Organization A

\section{Results and discussion}

We could recognize that Incident Management is a business process as any other. Therefore, BPM lifecycle is able to assist in its improvement, since the process identification and its variations (according to criticality, for example) to its monitoring \& control and redesign.

First result was discovery of actual Incident Management process by extracted data. From 5-steps process (Open, Assignment, Work in Progress, Resolved and Closed) we had taken statistics to measure time performance for each transition, and answered research question (i). Transition "Open to Assignment" was the bottleneck, the one causing more impact to resolution time. After mining incident event logs, we found that Organization A is taking, in average, more than double time to assign proper support team than to actually solve an incident. This shows also that stakeholder could discard transition "Resolved to Closed", that is, this transition not critical as the majority of this transition was handled automatically.

Incident Manager for Organization A had explained us that first level support uses knowledge articles to understand what is being requested by user and for what team should be assigned to solve it. He had also informed that many times there is no information, or information in not updated about support teams for each service, and because of that, first level support can commit assignment errors. Then we built a 
GQM+Strategies grid to organize and propose strategies and derive in questions, goals and metrics to be used by Organizational A in order to have a better control of Incident Management process and take proper actions to reach organization goal (Figure 4).

Selection of most appropriate metrics to be used is not a trivial task and is the success key for effective control and adherence to quality and process performance goals. This approach can support metrics selection activity required for Measurement and Analysis (CMMI-SVC level 3). By only using incident activities logs and interviewing Incident manager, we were able to (i) discover all possible process flows, (ii) find the process flow that is more commonly followed, (iii) find transition time between steps that is taking longer, and (iv) derive organization goal in strategies and metrics to improve quality of service.

\section{CONCLUSION AND FUTURE WORK}

In this paper we aimed to use BPM, BPI and Process Mining to discover Incident Management process by event logs and find bottleneck for incident resolution time, in order to support selection of metrics to attend organization goals and quality of services improvement. We identified areas that allow us to collaborate and contribute on a mutual basis Business Process Management and Quality of Services. Our objective was to link the theory and practical approach to generate knowledge and enable decision-making in one of these areas using Business Process Intelligence and IT Services Maturity Levels. The result would be the recommendations for the appropriate service and capacity model to support mining in IT operations. Through this collaboration, we expect to improve resources to support this initiative. We had obtained evidences that Business Process Management (BPM) and Business Process Intelligence (BPI) can be combined to support services maturity models. Also, we point there is little discussion about this aspect and that proposed approach can be conveniently and efficiently applied.

This case study can be modeled as a new method to be reproduced in order to select metrics by process bottleneck discovery and support root cause analysis for problems. Also, it shows we can generate knowledge about the process to support quality of services.

We can separate future work in two phases. First phase involves investigating methodological aspects: what, how and where to apply BPM and BPI techniques in service processes areas. Second phase involves researches that can help to quantify and to qualify the application of first phase in real situations. This will encompass analysis of case studies and interviews with experts. Dynamics within two phases could identify trends to improve IT solutions application within organizations and reduce required time for an organization to achieve a measurement process of IT services maturity models as well as the cost to maintain this level.

This is the first step we have taken in a path we argue is important to be followed. We hope to debate and envision new opportunities for action and believe the two areas converge from an academic and industrial point of view. The result will be the ability of increasing technological information efficiency to provide better solutions to organizations and society.

\section{ACKNOWLEDGMENT}

Authors would like to thank the financial support granted by FAPERJ (project E-26/110.438/2014).

\section{REFERENCES}

[1] Dumas, M., La Rosa, M., Mendling, J., Reijers, H.A. Fundamentals of Business Process Management, Springer, Berlin, Germany (2013)

[2] Castellanos, M., K.A.d. Medeiros, J. Mendling, B. Weber, and A.J.M.M. Weitjers, Business Process Intelligence, in Handbook of Research on Business Process Modeling, J. J. Cardoso and W.M.P. van der Aalst, Editors. 2009, Idea Group Inc. p. 456-480 (2009)

[3] Grigori, D., Casati, F., Catellanos, M, Dayal, U., Sayal, M., \& Shan, M. Business Process Intelligence. Computer in Industry, 53(3),321343.(2004)

[4] Aalst, W.M.P., van der, et al.: Process Mining Manifesto. Technical Report, IEEE Task Force, (2011)

[5] Process Mining Framework, http://www.processmining.org/prom/start (2014)

[6] Extensible Events Stream, http://www.xes-standard.org/ (2012)

[7] Ceurs Workshop Proceedings, Business Process Intelligence Challenge 2013, http://ceur-ws.org/Vol-1052/ (2013)

[8] CMMI INSTITUTE. "CMMI-SVC - Capability Maturity Model Integration for Services". http://cmmiinstitute.com/resource/cmmi-forservices-version-1-3/ (2010)

[9] Florac, W. A., Carleton, A. D., Measuring the Software Process: Statistical Process Control for Software Process Improvement, Addison Wesley (1999)

[10] ITIL Version 3 Service Design. Office of Government Commerce (OGC) (2007)

[11] ISO - International Standard Organization "ISO/IEC 20.000-2: Information Technology - Service Management - Part 2: Code of practice" - Switzerland. (2012)

[12] Information Systems Audit, et al. COBIT Five: A Business Framework for the Governance and Management of Enterprise IT. ISACA (2012)

[13] Niessink, F., Clerc, V., Tijdink, T., Vliet, H. The IT Service Capability Maturity Model - IT Service CMM, version 1.0RC1. (2005)

[14] Process Mining and Automated Process Discovery Software for Professionals - Fluxicon Disco, http://fluxicon.com/disco/ (2014)

[15] Solingen, R., Berghout, E. "The Goal Question Metric Method: A Practical Guide for Quality Improvement of Software Development" McGraw-Hill (1999)

[16] Franke, U., Holm, H., Konig, J., The Distribution of Time to Recovery of Enterprise IT Services - IEEE Transactions on Reliability (2014)

[17] Ferreira, D., Silva, M. Using Process Mining for ITIL Assessment: A Case Study with Incident Management. Proceedings of 13th Annual UKAIS Conference (2008)

[18] Mieke, J.; Lybaert, N.; Vanhoof, K.: Business Process Mining for Internal Fraud Risk Reduction: Results of a Case Study. Induction of Process Models (2008)

[19] Recker, J. "Scientific Research in Information Systems A Beginner's Guide". Springer ISBN 978-3-642-30048-6 (2013)

[20] Trinkenreich, B., Santos, G., Barcellos, M.. "Metrics to Support IT Service Maturity Models - A Systematic Mapping Study", 17th International Conference on Enterprise Information Systems (ICEIS), Barcelona, Spain (2015)

[21] Basili, V., Trendowicz, A., Kowalczyk, M., Heidrich, J., Seaman, C., Münch, J., Rombach D., "Aligning Organizations Through Measurement - The GQM+Strategies Approach”. Springer (2005)

[22] Davenport, T. "Process Innovation: Reengineering Work Through Information Technology". Harvard Business Press (2013)

[23] Niessink, F., Vliet, H., "Software Maintenance from a Service Perspective". Journal of Software Maintenance. v12 p.103-120 (2000) 